\title{
Model Hessian for accelerating first-principles structure optimizations
}

\author{
M. V Fernández-Serra, ${ }^{1,2}$ Emilio Artacho ${ }^{1}$, and José M. Soler ${ }^{2}$ \\ ${ }^{1}$ Department of Earth Sciences, University of Cambridge, Downing Street, Cambridge CB2 3EQ, UK \\ ${ }^{2}$ Depto. de Física de la Materia Condensada, C-III, Universidad Autónoma, 28049 Madrid, Spain
}

(Dated: September 19, 2017)

\begin{abstract}
We present two methods to accelerate first-principles structural relaxations, both based on the dynamical matrix obtained from a universal model of springs for bond stretching and bending. Despite its simplicity, the normal modes of this model Hessian represent excellent internal coordinates for molecules and solids irrespective of coordination, capturing not only the long-wavelength acoustic modes of large systems, but also the short-wavelength low-frequency modes that appear in complex systems. In the first method, the model Hessian is used to precondition a conjugate gradients minimization, thereby drastically reducing the effective spectral width and thus obtaining a substantial improvement of convergence. The same Hessian is used in the second method as a starting point of a quasi-Newton algorithm (Broyden's method and modifications thereof), reducing the number of steps needed to find the correct Hessian. Results for both methods are presented for geometry optimizations of clusters, slabs, and biomolecules, with speed-up factors between 2 and 8 .
\end{abstract}

PACS numbers: 02.60.Pn, 31.15.-p, 31.15.Ar, 71.15.-m, 71.15.Nc

The exponential growth of computer power together with recent methodological advances have opened firstprinciples electronic-structure calculations to systems of

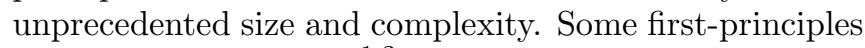
linear-scaling methods 1 (methods for which the required computer resources scale linearly with system size) already allow calculations involving a few thousand atoms. In many cases, the first problem to solve when facing a large and complex system is the first-principles determination of its structure by means of some energy minimization algorithm. As pointed out by Goedecker et al. 3 , the linear scaling achieved for each ab initio force evaluation degrades to a higher order scaling for the structure determination, since the number of force evaluations increases with system size. This is due to the increasing ill-conditioning of the structural optimization with larger system sizes, which has become the main bottleneck in the ab initio prediction of structures for the sizes treatable now.

In the case of conjugate gradients, for example, the number of evaluations is proportional to the condition number, defined as the ratio between the highest and the lowest non-zero curvatures of the energy landscape around the sought minimum. In any solid system, in the limit of large sizes, the lowest non-zero frequency is inversely proportional to a characteristic side length of the system, given the linear dispersion relation of long wavelength acoustic phonons. The condition number and thus the number of relaxation steps contributes an additional overall scaling factor of between $N^{1 / 3}$ an $N$, depending on the system shape. A clever solution 3 is to use the known (or approximate) macroscopic elastic properties to relax the long-wavelength low-frequency modes. However, with an increasing number of atoms, there is also an increase in the complexity of the system and of the different kinds of low frequency modes. In many complex systems of enormous importance (polymers, biomolecules, glasses, to name a few) there are low-frequency modes that do not correspond to any long-wavelength continuum limit.

This "complexity" is best characterized within the theory of rigidity 4 and its floppy modes. Their frequencies are very low since they do not involve the stretching, bending, or torsion of any particular bond, but their wavelengths can be very short, in many instances the modes being localized. A system with floppy modes is therefore ill-conditioned for relaxation. Even if these floppy modes do not strictly enter scaling arguments, they do spoil the structural optimizations of complex systems. The language introduced naturally in rigidity theory is that of internal coordinates: bond lengths, bond angles, and bond torsion angles. Internal coordinates are quite popular in chemistry and have indeed proven to be more efficient than Cartesian foordinates for the optimization of molecular systems. They are weaker, however, for large condensed systems since, on one hand, they do not address the acoustic ill-conditioning and, on the other hand, they are more complicated to handle for high coordinationst.

It is important to stress that avoiding ill conditioning requires a non-pathological identification of the low frequency modes, more than a very realistic description of the dynamical matrix. Realistif empirical potentials, no matter how good or universale, provide good dynamical matrices close to the minimum but may yield negative curvatures away from it. We present here a simple and natural way of addressing both sources of ill conditioning (acoustic and floppy) on the same footing. It constructs a positive-definite dynamical matrix from a universal model of springs for bond stretching and bending, and uses it to improve the geometry relaxation in two alternative ways, which correspond to two popular minimization methods.

Model Potential. The proposed Hessian model is based on a simple bond bending and stretching potential de- 
fined for any system at the given coordinates $\mathbf{r}_{i}^{0}$ as

$$
\begin{gathered}
U=\frac{1}{2} \sum_{i<j} k_{i j}^{s}\left(r_{i j}-r_{i j}^{0}\right)^{2}+\frac{1}{2} \sum_{i<j<l} k_{i j l}^{b}\left(\theta_{i j l}-\theta_{i j l}^{0}\right)^{2}, \\
k_{i j}^{s}=A\left(\frac{R_{i}+R_{j}}{r_{i j}^{0}}\right)^{8}, \\
k_{i j k}^{b}=B \sqrt{k_{i j}^{s} k_{j k}^{s}} r_{i j}^{0} r_{j k}^{0}
\end{gathered}
$$

where $R_{i}$ and $R_{j}$ are the covalent radii of the atoms connected 3 . The sums in Eq. (1) are limited to neighbors within $6 \times \max _{i}\left[R_{i}\right]$ The proportionality constant $A$ in Eq. (2) is irrelevant for the the first relaxation method described below but it does affect the second one (very moderately). Its value $A=3.0 \times 10^{5} \mathrm{eV} / \AA^{2}$ has been defined universally by adjusting the bulk modulus of cubic diamond, the largest known in nature. Albeit arbitrary, a large constant ensures small initial atomic displacements and thus the stability of the relaxation. The power 8 is also arbitrary but reasonable and has been chosen after some numerical tests. The relative magnitude of the bending and stretching constants has also been arbitrarily chosen as $B=1 / 10$ after some tests. The introduction of the even weaker torsional forces in the potential could bring further benefits and will be explored in later works.

The Hessian is evaluated at the minimum of the potential, which is defined by Eq. (1) to be at the given (initial) coordinates of the system to be relaxed. A positive definite Hessian is thus guaranteed. The model has no system-dependent parameters and is completely universal. Despite its simplicity, it captures qualitatively the separation between high-frequency stretching modes and lower-frequency bending and torsion modes. It also yields naturally the long wavelength acoustic modes, thus combining the main advantages of the methods of internal coordinates 5 and of elastic modes 3 .

Preconditioned conjugate gradients. The problem of ill conditioned minimization can be seen as the difficulty to find the way to the minimum along a gently sloped but narrowly shaped valley. The problem can be quantified by the condition number, the ratio $\omega_{\max }^{2} / \omega_{\min }^{2}$ between the curvatures across and along the valley or, in a higher dimensional space, between the largest and smallest curvatures. The number of steps in the minimization process increases with growing condition number, the precise scaling depending on the particular algorithm, quadratic for steepest descent and linear for conjugate gradients, for example.

The number of evaluations of the ab initio forces (the gradient) can be thus reduced by a transformation of coordinates such that the curvatures in the new space give a smaller condition number. The effort required for the coordinate transformations is negligible compared with that of calculating the ab initio forces. Such preconditioning can be accomplished by using a priori knowledge about the system curvatures.

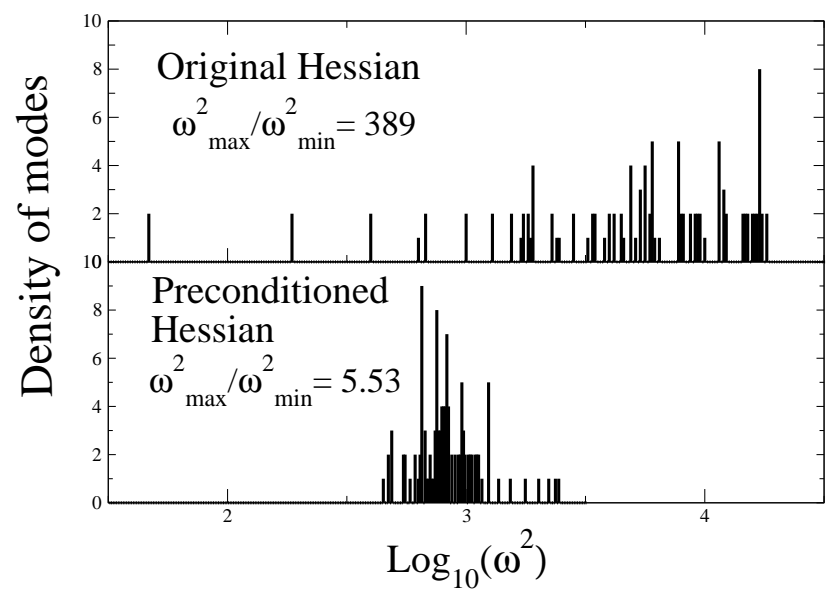

FIG. 1: Histogram of eigenvalues $\omega^{2}$ of the Hessian (matrix of second derivatives) of a 247-atom gold slab made of ten layers of 25 atoms each, with-three vacancies. simulated with an embedded atom potentialld. Upper panel: using standard Cartesian coordinates. Lower panel: using transformed coordinates, for which the eigenvalues of our model Hessian become all equal. The condition number (ratio between largest and smallest eigenvalues) is given for each case. The units of $\omega$ are $\mathrm{cm}^{-1}$.

Our preconditioning procedure requires an initial diagonalization of the Hessian matrix of the model defined in Eq. (1):

$$
H_{i j}=\frac{\partial^{2} U}{\partial x_{i} \partial x_{j}} .
$$

The ab initio forces $\mathbf{F}$ are then projected over the normal modes $\mathbf{n}$ of that Hessian matrix and divided by the 'frequencies' $\omega_{n}$ (the square root of the eigenvalues):

$$
f_{n}=\frac{\mathbf{F} \cdot \mathbf{n}}{\omega_{n}}
$$

A conventional conjugate gradients routine is then used to minimize the ab initio energy as a function of the new coordinates:

$$
y_{n}=\omega_{n}(\mathbf{x} \cdot \mathbf{n})
$$

where $\mathbf{x}$ are the Cartesian coordinates.

The initial diagonalization represents an unimportant computational effort for the systems treated nowadays with first-principles techniques. In the future, however, this foreseeable limiting step will have to be given some more thought. For example, the mentioned rigidity theory could be further exploited, given its ability of predicting subsets of floppy atoms versus rigid bits of the overall structure.t

Fig. 11 shows the reduction in spectral width and condition number for a 10-layer old slab, simulated with an embedded atom potentiale. This many-body potential has been chosen for some of our tests because the efficiency of our minimization methods, in terms of the 
number of iterations, does not depend on the specific form of the interactions. In the new coordinates the condition number is reduced by a factor 70 , proving that the model potential represents a good initial approximation to the real one.

Quasi-Newton method. Variable-metric method,11, 12 minimize the energy by applying

$$
\delta \mathbf{x}=-H^{-1} \delta \mathbf{F}=H^{-1} \mathbf{F}
$$

where $H$ is the Hessian and $\delta \mathbf{F}=-\mathbf{F}$ is the desired change of the forces. Given the exact Hessian of a perfectly harmonic function, one step would suffice to find its minimum. Since the potential is generally not harmonic and the Hessian is unknown, an iterative process is followed in practice, starting with a trial Hessian, moving according to (7) and updating the Hessian afterwards so that it obeys (7) for the true change of the force found. Since no information is generally known about the Hessian, a unity matrix is used as the initial $H^{-1}$. We simply change this to the inverse of our model Hessian and then use a standard variable-metric method 12 . Again, the fact that the model Hessian captures nontrivial low-frequency modes allows the iterative learning process to save many steps to find out about them (the ill-conditioning affects this kind of methods as well). The initial inversion of the model Hessian represents a very small overhead compared with the evaluation of ab initio forces.

In addition, Broyden-like methods lend themselves nicely to hierarchical approaches whereby lower quality relaxations can feed the Hessian for higher quality ones. For example, one can perform a relaxation with a small basis set for the electronic structure, starting from our model Hessian, and use the resulting ab initio inverse Hessian to launch a finer relaxation with a better basis set. We will explore this approach in a later work.

The two methods have been applied to systems of different character and complexity, namely, to metal clusters and slabs, and a biomolecule. They constitute quite extreme examples of complex systems. On one hand, two systems with high coordinations and a non trivial energy landscape 13 On the other, a low-coordination molecule with floppy modes.

Table I shows results for the relaxation of two gold systems, simulated with the embedded atom potentialed: a ten-layer crystalline slab and an amorphous 77-atom cluster. The number of iterations needed to reach the minimum are given as a function of its distance from the initial coordinates, chosen at random within a window of $\pm \delta x^{0}$ from the relaxed structure. Our two methods are compared with (i) regular CG, (ii) CG preconditioned with the exact Hessian, and (iii) Broyden's method starting from the identity matrix. The exact Hessian is calculated at the minimum because otherwise it soon develops negative eigenvalues, whereas the model one is evaluated at the starting point, since it is always positive definite.

The table shows that our model Hessian improves considerably the efficiency of both the CG and the mod- ified Broyden methods. As expected, the efficiency of the Broyden-like schemes is superior when starting close to the minimum, though this advantage decreases with initial separation from the harmonic basin. It is also interesting the comparison of our preconditioned CG with the one using the exact Hessian at the minimum (which is generally not available in practice, of course). The latter is extraordinarily efficient when started well within the harmonic basin, but it deteriorates very rapidly with distance, making it not much better than our method in practice. We do not, therefore, expect a better performance if using realistic empirical potentials instead of our universal model.

Fig. 2 shows the convergence in energy, forces, and atomic positions, for the ten-layer gold slab with the different methods discussed. Within CG, the convergence is slower during the first steps with the preconditioned method for the energy and the forces, but not for the coordinates. This is because CG responds with larger displacements to the high curvature modes, which dominate the energy drop in the initial stages. The situation is reverted soon, however, and the overall efficiency is clearly better for preconditioned CG.

The modified Broyden method with our model Hessian initialization is most efficient for small displacements from the minimum, accelerating the convergence by factors of 3 to 8 . In this and other systems, we have generally found that the Broyden method is extremely effective whenever it starts well within the harmonic basin. However, it is rather sensitive to other effects, as, for instance, the space inhomogeneity introduced by the grid used to integrate the Hartree and exchange-correlation energies in our ab initio methodi. On the other hand, the preconditioned CG method appears to be more robust against this kind of effects.

Fig. 3 shows the convergence for a piece of a double helix of DNA with two base pairs (134 atoms). The forces in this case were calculated using ab initio densityfunctional theory, norm-conserving pseudopotentials and a basis set of numerical atomic orbitals. 1 The preconditioned conjugated-gradient method represents a much better option for relaxing this molecular system. Low energy modes not included in the acoustic branch are responsible of the ill-conditioning on the relaxation of these sort of structures and the model takes account of them, improving the convergence by a factor of two.

We have developed two methods for accelerating first principles structure relaxations, based on a classical and universal model Hessian. Drastic improvements in overall efficiency are achieved, reducing the number of minimization steps by factors of 2 to 8 in the cases studied. Of the two methods presented, the Broyden method is more efficient when sufficiently close to the minimum, while the preconditioned CG method is more robust when the energy landscape is far from harmonic. 
TABLE I: Number of iterations needed to reach a force tolerance of $10^{-6} \mathrm{eV} / \AA$ in a ten-layer crystalline slab and a 77 -atom cluster of gold, simulated with an embedded atom potential. The initial atomic coordinates were randomly displaced from the equilibrium geometry in an interval of $\pm \delta x^{0} . F_{\max }^{0}$ is the maximum initial atomic force. The conjugate gradient method was used with conventional Cartesian coordinates (CG) and with preconditioned coordinates (PGC) that diagonalize the model Hessian (at the initial geometry) or the exact Hessian (at the minimum). The variable-metric Broyden method was used starting with the conventional unit-matrix Hessian and with our new model Hessian.

\begin{tabular}{|c|c|c|c|c|c|c|c|}
\hline System & $\begin{array}{l}\delta x^{0} \\
(\AA)\end{array}$ & $\begin{array}{c}F_{\max }^{0} \\
(\mathrm{eV} / \AA)\end{array}$ & CG & $\begin{array}{c}\text { PCG } \\
\text { Model H }\end{array}$ & $\begin{array}{c}\text { PCG } \\
\text { Exact } \mathrm{H}\end{array}$ & $\begin{array}{c}\text { Broyden } \\
\text { Unit H }\end{array}$ & $\begin{array}{l}\text { Broyden } \\
\text { Model H }\end{array}$ \\
\hline 250-atom & $10^{-4}$ & $2.13 \times 10^{-3}$ & 40 & 18 & 3 & 8 & 5 \\
\hline crystalline & $10^{-3}$ & $2.13 \times 10^{-2}$ & 100 & 29 & 7 & 15 & 9 \\
\hline \multirow[t]{2}{*}{ slab } & $10^{-2}$ & $2.14 \times 10^{-1}$ & 180 & 35 & 10 & 32 & 14 \\
\hline & $10^{-1}$ & 2.52 & 240 & 60 & 45 & 339 & 33 \\
\hline 77-atom & $10^{-4}$ & $3.03 \times 10^{-3}$ & 51 & 22 & 3 & 22 & 14 \\
\hline amorphous & $10^{-3}$ & $3.03 \times 10^{-2}$ & 72 & 40 & 6 & 29 & 19 \\
\hline \multirow[t]{2}{*}{ cluster } & $10^{-2}$ & $3.04 \times 10^{-1}$ & 101 & 53 & 9 & 36 & 25 \\
\hline & $10^{-1}$ & 3.25 & 167 & 90 & 50 & 140 & 27 \\
\hline
\end{tabular}

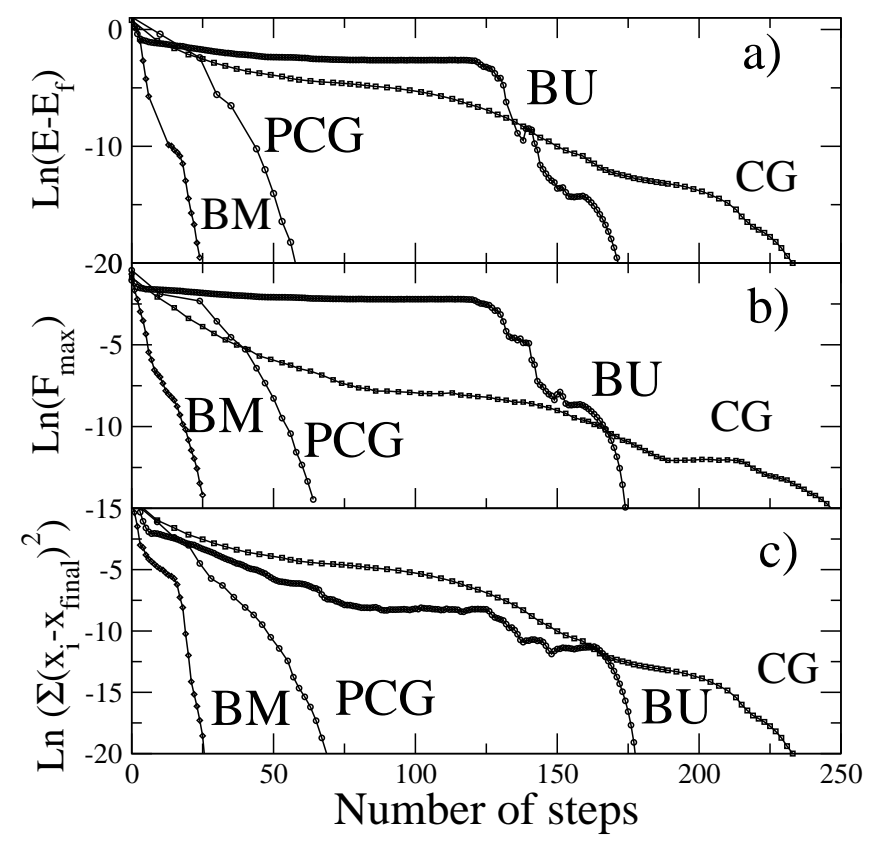

FIG. 2: Logarithmic convergence of the energy (a), forces (b), and coordinates (c), for a 10-layer gold slab with 247 atoms and three vacancies. The initial coordinates where randomly displaced by $\sim 0.2 \AA$ from the equilibrium geometry. Four different minimization methods were used: conventional conjugate gradients (CG); preconditioned conjugate gradients (PGC); Broyden's method starting from a unit Hessian matrix (BU); and Broyden's method starting from our model Hessian (BM).

\section{Acknowledgments}

This work was funded by the Spanish Ministerio de Ciencia y Tecnología under grant BMF2000-1312 and by the Fundación Ramón Areces. MFS acknowledges sup-

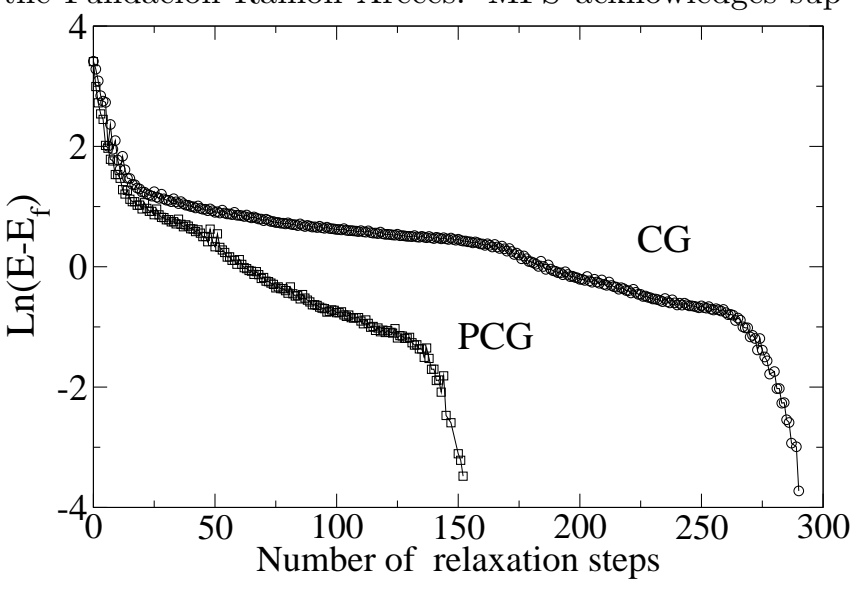

FIG. 3: Logarithmic convergence of the energy of a two-basepair DNA strand relaxed with conventional conjugate gradients (CG) and preconditioned conjugate gradients (PGC) methofs, using atomic forces obtained from density functional theoryt.

port from a Studentship from the Comunidad Autónoma de Madrid.
1 J. M. Soler, E. Artacho, J.D. Gale, A. García, J. Junquera, P. Ordejón, and D. Sánchez-Portal, J. Phys.: Condens. Matter 14, 2745-2779 (2002).

2 D. R. Bowler, T. Miyazaki, and M. J. Gillan, J. Phys.:
Condens. Matter 14, 2781-2798 (2002).

3 S. Goedecker, F. Lanon, and T. Deutsch, Phys. Rev. B 64, 161102 (2001).

4 J. C. Phillips and M. F. Thorpe, Solid State Comm. 53, 
699-702 (1985)

${ }^{5}$ P. Pulay, G. Fogarasi, F. Pang, J. Boggs, J. Am. Chem. Soc. 101, 2550 (1979).

6 Jon Baker, J. Chem. Phys. 105192 (1996).

7 J. Andzelm, R. D. King-Smith, and G. Fitzgerald, Chem. Phys. Lett. 335, 321-326 (2001)

8 www.webelements.com

9 A. K. Rappe, C. J. Casewit, K. S. Colwell, W. A. Goddard III, and W. M. Skiff, J. Am. Chem. Soc. 114, 10024-10035 (1992)
10 S.M. Foiles, Phys. Rev. B 32, 3409 (1985); M. S. Daw and M. I. Baskes, Phys.Rev. B 29, 6443 (1984).

11 W. H. Press, S. A. Teukolsky, W. T. Vetterling and B. P. Flannery Numerical Recipes, Cambridge Univ. Press, 1992

12 D. Vanderbilt and S. G. Louie, Phys. Rev. B 30, 6118 (1984).

13 J. M. Soler, M. R. Beltrán, K. Michaelian, I. L. Garzón, P. Ordejón, D. Sánchez-Portal, and E. Artacho, Phys. Rev. B 61, 5771-5780 (2000) 